\title{
Caregivers' appraisals of patients' involuntary hospital treatment: European multicentre study
}

Domenico Giacco, Andrea Fiorillo, Valeria Del Vecchio, Thomas Kallert, Georgi Onchev, Jiri Raboch, Anastasia Mastrogianni, Alexander Nawka, Tomasz Hadrys, Lars Kjellin, Mario Luciano, Corrado De Rosa, Mario Maj and Stefan Priebe

\section{Background}

Mental health policies emphasise that caregivers' views of involuntary psychiatric treatment should be taken into account. However, there is little evidence on how caregivers view such treatment.

\begin{abstract}
Aims
To explore caregivers' satisfaction with the involuntary hospital treatment of patients and what factors are associated with caregivers' appraisals of treatment.
\end{abstract}

\section{Method}

A multicentre prospective study was carried out in eight European countries. Involuntarily admitted patients and their caregivers rated their appraisal of treatment using the Client Assessment of Treatment Scale 1 month after admission.

\section{Results}

A total of 336 patients and their caregivers participated. Caregivers' appraisals of treatment were positive (mean of 8.5 on a scale from 0 to 10) and moderately correlated with patients' views. More positive caregivers' views were associated with greater patients' symptom improvement.

\section{Conclusions}

Caregivers' appraisals of involuntary in-patient treatment are rather favourable. Their correlation with patients' symptom improvement may underline their relevance in clinical practice.

\section{Declaration of interest \\ None.}

Recent mental health policies emphasise the need for psychiatric services to involve family caregivers in treatment planning, to help them cope with practical and psychological difficulties related to their role as caregivers and also to consider their views in the evaluation of treatment. ${ }^{1,2}$ Involving caregivers is particularly relevant in the challenging context of involuntary hospital treatment, which can lead to high levels of distress among all parties involved including caregivers, and requires good collaboration between clinicians and caregivers to optimise treatment and further care planning. ${ }^{3}$ Only a small number of studies have been conducted on caregivers' views of involuntary hospital treatment. ${ }^{3,4}$ All of them used qualitative methods with rather small samples. In these studies caregivers expressed high levels of dissatisfaction and complained they received too little information and poor guidance from staff and were insufficiently involved in treatment decisions, particularly discharge planning. ${ }^{3,4}$ Studies using quantitative methods for assessing relatives views on in-patient care focused on burden, perceived coercion and need for support and information rather than on appraisals of the psychiatric treatment received by the patients. ${ }^{5-9}$

A European multicentre study was conducted as part of the European Evaluation of Coercion in Psychiatry and Harmonization of Best Clinical Practice (EUNOMIA) project. The general aims of the EUNOMIA project were to explore both practice and outcomes of involuntary hospital treatment across a range of European countries. The outcomes were assessed using both observer-rated standardised instruments and patientreported measures. ${ }^{10-14}$ Previous papers reported that 3 months after admission $63 \%$ of patients felt that the admission had been right, ${ }^{11}$ that symptom levels showed on average a significant but limited improvement, ${ }^{13}$ and that a reduction of positive symptoms was associated with less perceived coercion of patients. ${ }^{14}$ Given the important role of caregivers in the process of involuntary treatment, the project did not only assess the views of patients, but also those of caregivers. The findings are reported in this paper. Specifically, we studied how satisfied caregivers were with the treatment that their ill relatives received in the hospital. Using quantitative methods to assess caregivers' appraisal of treatment, we further explored which sociodemographic characteristics of the caregivers, types of relationships between patient and caregiver, sociodemographic and clinical characteristics of the patients and clinical treatment outcomes were associated with more or less positive appraisals. The study was conducted in eight countries: Bulgaria, Czech Republic, Germany, Greece, Italy, Poland, Slovakia and Sweden.

\section{Method}

\section{Design and participants}

At study sites in eight countries legally involuntarily admitted patients and their main caregivers were recruited. Patients were recruited and interviewed within the first week of admission. Data on the characteristics of the hospitals and other mental health services at each site have been described in detail elsewhere. ${ }^{10-14}$

\section{Inclusion and exclusion criteria}

Inclusion criteria for patients were: involuntary admission to psychiatric hospital according to the national legislation; age between 18 and 65 years; written informed consent obtained before the clinical assessment; not admitted to a special unit for only forensic patients or patients with substance dependence; not admitted to a special treatment programme for eating disorders; no main clinical diagnosis of dementia; not yet included in the study; not transferred to one of the participating hospitals from another hospital; currently living in the catchment area of the participating hospital(s); sufficient knowledge of the national language; and living with other people in the same household. Caregivers were included if, among all the people living with the patient, they were the one spending most of the time with the patient, and were also able to provide informed consent. 


\section{Measures and procedures}

Sociodemographic characteristics of caregivers and patients and the type of the relationship between the patient and caregiver were recorded on a structured questionnaire. Data on previous patient hospital admissions and the clinical discharge diagnosis according to the diagnostic criteria of ICD- $10^{15}$ were recorded anonymously for all patients admitted to the centres participating in the EUNOMIA study within the study period; these data were available in an aggregated form from the hospital statistics.

Patients and caregivers rated their appraisal of treatment using the Client Assessment of Treatment Scale (CAT), which consists of seven items addressing different aspects of hospital treatment. ${ }^{16}$ The patient version of the scale has been widely used in research. In patients receiving involuntary hospital treatment, a high internal consistency (Cronbach's $\alpha=0.90)^{16}$ and a factorial validity with an invariant one-factor model have been reported across samples in different countries (UK, Spain and Bulgaria). ${ }^{17}$ To obtain the views of caregivers, the same seven items were used with modified wording to capture the view of the caregiver instead of that of the patient (Table 1). All items are rated on 11-point scales (i.e. 0 to 10 ) with higher scores indicating more positive views. The patient's psychiatric symptoms were assessed on the 24 -item version of the Brief Psychiatric Rating Scale (BPRS). ${ }^{18}$

Patients were recruited from between one and five hospitals per country. Patients who were recruited to the study and lived with a caregiver were asked for permission for the researcher to contact the caregiver. If the patient consented, caregivers were contacted and invited to an interview, which usually took place at the hospital. Researchers who were independent from the treatment team approached and interviewed patients and caregivers. Patients' symptoms were assessed at baseline and 4 weeks after admission. Patients' and caregivers' appraisals of treatment were also obtained at the interview after 4 weeks. All researchers on the study were trained in the required assessments and achieved an interrater reliability of Cohen's kappa $(\kappa)=0.90$ on the BPRS.

\section{Statistical analysis}

Descriptive statistics were used to analyse sociodemographic and clinical characteristics of patients, sociodemographic characteristics of caregivers and the treatment appraisals of caregivers and patients. The internal consistency of the CAT for caregivers and the CAT for patients was measured by means of Cronbach's alpha test. The correlation between the scores from the CAT for caregivers and that from patients was evaluated using the Pearson's test for bivariate correlations. The scores from caregivers and patients were compared using the $t$-test for paired samples. Univariable linear regression models were computed to assess the univariable associations of the characteristics of caregivers and patients with caregivers' appraisal of treatment. As potentially relevant characteristics we tested age and gender of caregivers; the type of relationship between patient and caregiver (i.e. being patients' parents, spouses/partners, brothers/sisters, sons/daughters or other type of relation); patients' age, gender, previous hospital admission (no, 0; yes, 1), clinical diagnosis (grouped in three categories: psychotic disorders, mood disorders and other disorders), symptom levels at baseline and 4-week follow-up (BPRS total score) of the patients. Variables that were significantly associated with caregivers' appraisal $(P<0.05)$ were then entered in a multivariable linear regression model. In this analysis, the symptom levels at follow-up were adjusted for baseline symptoms so that they reflected clinical improvement during the 4-week treatment period. Multivariable regression also adjusted for the influence of country. In a sensitivity analysis, the multivariable linear regression model produced was tested in a subgroup of caregivers of patients with schizophrenia and related disorders (ICD-10: F20-29). Statistical analyses were performed using the Statistical Package for Social Sciences (SPSS), version 18.0 for Windows. The level of statistical significance was set at $P<0.05$ for all analyses.

\section{Results}

\section{Characteristics of patients and caregivers}

Table 2 shows the recruitment of patients and caregivers for each country. Out of 3230 patients eligible for the study, 2171 (67.2\%) were contacted and asked to participate, and 1552 (48.0\%) were recruited to the study in the eight participating countries. Out of this group of recruited patients, 574 patients (36.9\%) lived with a caregiver who represented the sample of potentially eligible caregivers in this study. In 399 cases $(69.5 \%$ of all potentially eligible caregivers), the caregiver was contacted and consented to participate. Only 336 of them turned out to be the main caregiver, who spent more time with the patient than other people in the same household. Thus, 336 patients and their caregivers were included, which represents $58.5 \%$ of all potentially eligible caregivers.

The 336 included patients were predominantly male (56.3\%), with a mean age of 38.9 years (s.d. $=11.5$ ); $66 \%$ of them had been previously admitted to psychiatric hospital treatment. The clinical discharge diagnoses were schizophrenia or related disorder $(n=213,63.4 \%)$, mood disorder $(n=47,14 \%)$, substance use disorder $(n=40,11.9 \%)$, disorders of adult personality and behaviour $(n=20,6 \%)$, organic brain disorder $(n=7 ; 2.1 \%)$, anxiety disorder $(n=5,1.5 \%)$ and other disorders $(n=4,1.2 \%)$. Six $(1.8 \%)$ of the patients had been discharged at follow-up.

Caregivers were predominantly female $(62 \%)$ and had a mean age of 50.9 years $($ s.d. $=17.6)$. They were the parents $(n=156$, $46.4 \%)$, spouses $(n=102,30.4 \%)$, brothers or sisters $(n=36$, $10.7 \%)$, sons or daughters $(n=24,7.1 \%)$ of the patients; 11 (3.3\%) were another type of relation (grandfather/grandmother, uncle/aunt) and 7 (2.1\%) reported to be friends of the patients.

\section{Caregivers' and patients' appraisals of treatment}

The Cronbach's alpha for the seven items of the CAT was 0.87 for caregivers and 0.91 for patients. The correlation between the CAT for caregivers and patients was $r=0.530(P<0.0001)$. The mean score of the caregivers on the CAT was $8.5($ s.d.=1.4) and that of the patients 7.7 (s.d. $=2.1$ ). The difference was statistically significant $(t=4.926, P<0.0001)$. The mean scores for each item of the CAT for caregivers and patients are reported in Table 1. The mean scores for caregivers ranged from 7.8 ('Do you believe his/her relations with other staff members are pleasant or unpleasant for him/her?') to 8.5 ('Do you believe your relative is receiving the right medication for him/her?'). For the subgroup of caregivers of patients with schizophrenia and related disorders the scores were similar to those of the total sample, with a CAT mean score of 8.5 (s.d.=1.6), whereas for patients in this subgroup appraisals of treatment were less positive than those of the other diagnostic groups $(7.4 \quad($ s.d.=2.2) v. $8.1 \quad$ (s.d.=2.6), $F=5.644, P<0.05)$.

\section{Association of caregivers' and patients' characteristics with caregivers' appraisal of treatment}

Table 3 shows the univariable associations of caregivers' and patients' characteristics with caregivers' appraisal of treatment. 
Table 1 Mean caregiver and patient scores on the Client Assessment of Treatment Scale (CAT)

$\begin{array}{lc}\text { CAT items } & \text { Mean (s.d.) } \\ \text { Caregivers } & 8.1 \text { (2.4) } \\ \text { 1. Do you believe your relative is receiving the right treatment/care for him/her at the hospital? } & 8.1 \text { (2.6) } \\ \text { 2. Do you believe his/her therapist/case manager/keyworker understands him/her and is engaged in his/her treatment/care? } & 7.8(2.3) \\ \text { 3. Do you believe his/her relations with other staff members are pleasant or unpleasant for him/her? } & 8.5(2.1) \\ \text { 4. Do you believe your relative is receiving the right medication for him/her? } & 8.4(2.3) \\ \text { 5. Do you believe the other elements of treatment/care (e.g. occupational or group therapy) at the hospital are right for him/her? } & 7.9(2.6) \\ \text { 6. Do you believe he/she is feeling respected and regarded well at the hospital? } & 8.3(2.3) \\ \text { 7. Do you believe that the treatment/care your relative is receiving at the hospital is being helpful for him/her? } & 7.2(2.9) \\ \text { Patients } & 7.7(2.8) \\ \text { 1. Do you believe you are receiving the right treatment/care for you here? } & 8.0(2.2) \\ \text { 2. Does your therapist/case manager/keyworker understand you and is he/she engaged in your treatment/care? } & 7.1(3.1) \\ \text { 3. Are relations with other staff members here pleasant or unpleasant for you? } & 7.5(2.7) \\ \text { 4. Do you believe you are receiving the right medication for you? } & 7.8(2.6) \\ \text { 5. Do you believe the other elements of treatment/care here are right for you? } & 7.2(3.0) \\ \text { 6. Do you feel respected and regarded well here? } & \\ \text { 7. Has treatment/care here been being helpful for you? } & \end{array}$

\begin{tabular}{|c|c|c|c|c|c|c|c|c|c|}
\hline & Bulgaria & Czech Republic & Germany & Greece & Italy & Poland & Slovakia & Sweden & Total \\
\hline \multicolumn{10}{|l|}{ Patients } \\
\hline \multirow{3}{*}{$\begin{array}{l}\text { Eligible patients, } n \\
\text { Patients invited to participate, } \\
n \text { (\% of eligible patients) } \\
\text { Patients recruited, } \\
n \text { (\% of eligible patients) }\end{array}$} & 475 & 581 & 466 & 349 & 280 & 334 & 439 & 306 & 3230 \\
\hline & $372(78.3)$ & $341(58.7)$ & $221(47.4)$ & $248(71.1)$ & $213(76.1)$ & $252(75.5)$ & $311(70.8)$ & $213(69.6)$ & $2171(67.2)$ \\
\hline & $309(651)$ & $202(348)$ & $145(311)$ & $222(636)$ & $129(461)$ & $152(45.5)$ & $296(674)$ & 97 (317) & $1552(180)$ \\
\hline \multicolumn{10}{|l|}{ Patients living in close contact with } \\
\hline relatives, $n$ (\% of recruited patients) & $124(40.1)$ & $84(41.6)$ & $47(32.4)$ & $55(24.8)$ & $49(37.9)$ & $67(44.1)$ & $119(40.2)$ & $29(29.9)$ & 574 (36.9) \\
\hline \multicolumn{10}{|c|}{$\begin{array}{l}\text { Caregivers, } n \text { (\% of potentially } \\
\text { eligible caregivers) }\end{array}$} \\
\hline \multicolumn{9}{|l|}{ Caregivers agreeing to participate } & $399(69.5)$ \\
\hline the study & $47(37.9)$ & $36(42.9)$ & $37(78.7)$ & $34(61.8)$ & $48(97.8)$ & $57(85.1)$ & $68(57.1)$ & $9(31.0)$ & $336(58.5)$ \\
\hline
\end{tabular}

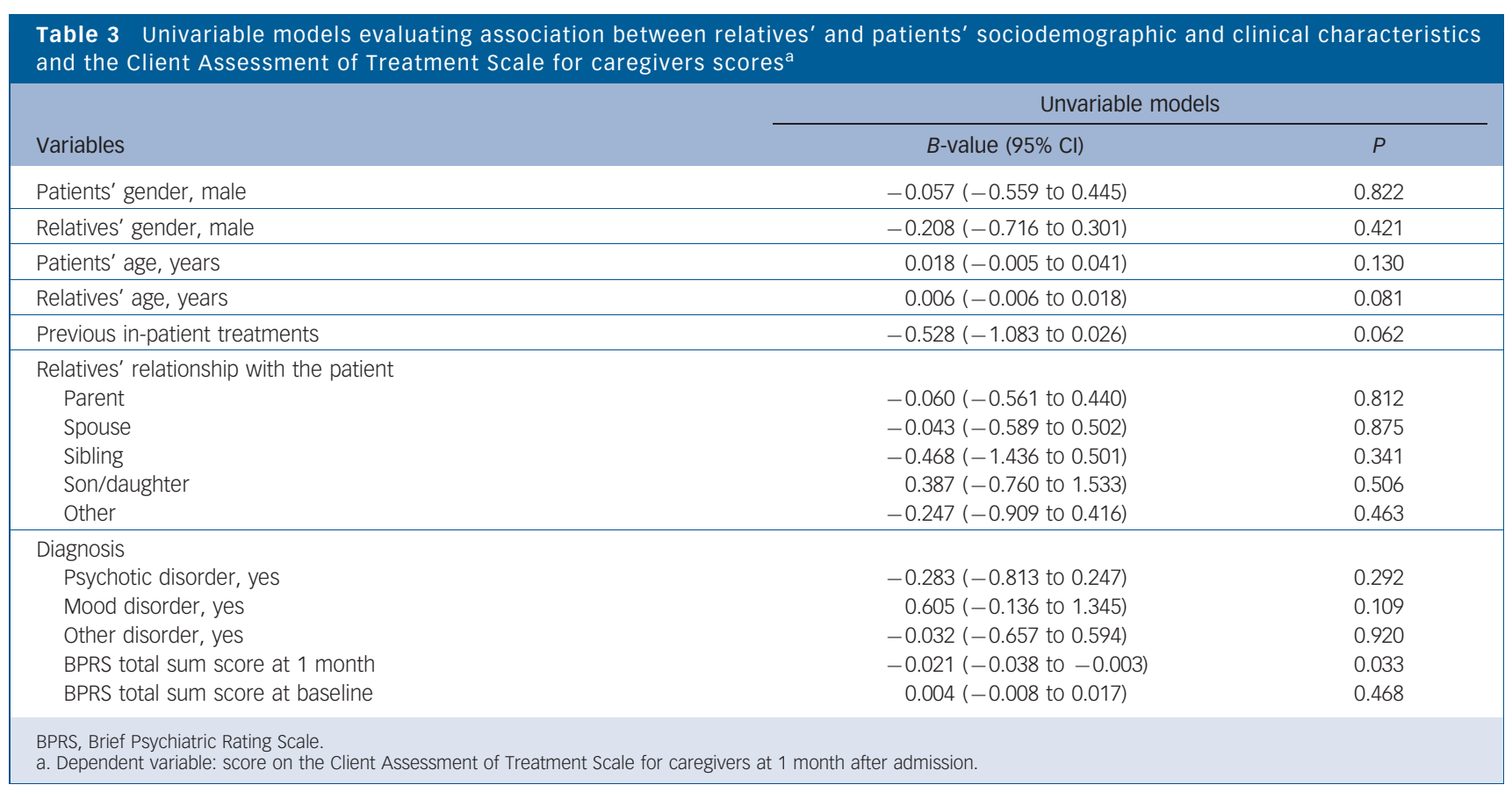

Sociodemographic characteristics of caregivers and patients, the type of relationship, previous patient hospital admissions, diagnosis and baseline symptom levels were not significantly associated with caregivers' appraisal of treatment. The only variable significantly correlated with caregivers' appraisal was the symptom level of patients at the 4-week follow-up. Caregivers of patients with lower symptom levels at 4 weeks tended to appraise treatment more positively. 
The multivariable analysis considered symptoms at follow-up as the only variable with a significant univariable association with caregivers' appraisal, and adjusted for symptoms at baseline (to reflect symptom improvement) and country. In this analysis, the association between symptoms at follow-up and caregivers appraisal was even more marked $(B$-value $=-0.037,95 \%$ CI -0.057 to $-0.017, P<0.001)$. A more favourable symptom change for patients during treatment is significantly linked with more positive treatment appraisals by caregivers. The results held true in a sensitivity analysis with caregivers of patients with schizophrenia and related disorders. The association between patients' symptom change and caregivers' appraisals was stronger than in the total sample $(B=-0.048,95 \%$ CI -0.073 to -0.024 , $P<0.001)$.

\section{Discussion}

\section{Main findings}

Overall, caregivers seem to view the involuntary hospital treatment of patients rather positively, with an average mean score of 8.5 on a scale of seven items, each of which has a maximum score 10. Their appraisal is more positive than that of the patients and moderately associated with it. Out of all variables tested as potentially influencing the caregivers' appraisal, only patients' symptoms after 4 weeks and, in particular, their symptom improvement were associated with caregivers' appraisal. When patients showed a more favourable symptom change after 4 weeks, caregivers tended to have a more positive view of treatment. This was found in the total sample as well as in the subgroup of patients with schizophrenia and related disorders.

\section{Strengths and limitations}

To our knowledge, this is the first study assessing caregivers' appraisal of involuntary hospital treatment in a systematic manner and using quantitative methods in a relatively large sample. The study was conducted in eight countries using a consistent methodology. The instrument used in the study to measure appraisal of treatment, the CAT, showed a good reliability in both versions (the patient version and the modified version for caregivers) despite having only seven items. Its brevity and easy use are likely to have contributed to the good response rate among caregivers. Further strengths of the study are that patients were assessed by trained researchers using standardised instruments and having good interrater reliability on the symptom ratings.

The study has three major limitations. First, the sample may be biased. We did not manage to recruit more than $48 \%$ of all eligible patients in the total baseline sample. Once patients were recruited and reported to have a caregiver, the inclusion rate of caregivers was better. In $58.5 \%$ of cases in which patients reported to live with a caregiver, the caregiver was interviewed. One can only speculate as to whether patients and caregivers with difficult relationships were less likely to participate in the research. It should be noted that only $36.9 \%$ of recruited patients reported to have a caregiver, and that therefore the patients in the final sample present only $10.4 \%$ of the total sample of eligible patients at baseline. As compared with the total sample of patients, most of whom did not live with a relative, the final number of included patients tended to stay longer in hospital. They also expressed a more positive appraisal of treatment than is commonly found in patients in involuntary hospital treatment. ${ }^{19,20}$ Second, the study assessed neither extent and type of care provided by caregivers to patients outside hospital treatment nor the frequency with which caregivers had visited patients in the hospital to gain a personal impression of the quality of the treatment. Third, caregivers' views of treatment were assessed only once after 4 weeks. It might be possible that, when hospital treatments last for a longer period, other factors such as relationships with staff and availability of rehabilitation programmes become more important in influencing caregivers' satisfaction, as it is the case during out-patient treatment. ${ }^{21}$

\section{Comparison with the literature}

Caregivers expressed high levels of satisfaction with all aspects of treatment covered in the questionnaire. Although there are no studies using similar methods with which to compare these findings, they appear to contrast with the results of qualitative studies that documented very negative experiences and substantial dissatisfaction of caregivers during involuntary hospital treatment of patients. ${ }^{3,4}$ There are three aspects that might explain this contrast. First, the samples in the qualitative studies were smaller and possibly more selective with convenience and purposive sampling. ${ }^{3,4}$ Thus, the negative views captured in these studies might have been influenced by a strong selection bias with a higher motivation of more discontent caregivers to participate and air their views in long interviews. Second, the qualitative studies addressed the experiences of caregivers linked to involuntary hospital treatment more widely. The interviews were not restricted to appraisals of how the patient was treated in the hospital as it is reflected in the CAT. They also covered aspects such as the admission procedure, the communication between clinicians and caregivers and discharge planning. Caregivers may be more dissatisfied with the support and information they receive from clinical staff during the period of the hospital admission than with the precise treatment that patients receive in the hospital. ${ }^{3,4,22}$ Finally, in qualitative studies caregivers were asked about their experiences in retrospect, i.e. after the discharge of the patient, whereas in this study they were interviewed when, in most cases, patients were still being treated in the hospital. Some previous studies suggest that caregivers tend to be careful about criticising services when they feel dependent on them. ${ }^{22,23}$ The appraisals captured in this study appear also more positive than those in a Swedish study ${ }^{7}$ that used a single item to assess the relatives' view of the quality of psychiatric services in general and found negative views in $40 \%$. Again, the relatives may have more positive views of the psychiatric hospital treatment as it was assessed in our study than on services in general.

Caregivers' appraisals were also positive in comparison to those found in studies on caregivers' satisfaction with treatment in out-patient and non-coercive in-patient settings. ${ }^{21-23}$ Patients who are involuntarily admitted to hospital treatment commonly have high symptom levels and challenging behaviour with risks to themselves or others before admission. In such a context, caregivers may experience high levels of burden ${ }^{24-29}$ and, therefore, appreciate more the treatment provided in a hospital and perceive the aspects of that treatment as rather positive.

It can be assumed that caregivers often base their judgement of the treatment on the descriptions and explanations of the patients. They may also share their own views with patients and influence how patients perceive treatment. Both processes may explain why caregivers' and patients' views of treatment are correlated. Yet, their views are not identical and the correlation of the appraisals is only moderate.

Patients show slightly lower levels of satisfaction with treatment than their caregivers. Patients can base their appraisal of treatment on the everyday lived experience of treatment on the ward. Caregivers do not have that direct experience. They can assess treatment only through observations during visits and 
reports by patients and clinicians. Also, being treated involuntarily in a hospital can be stressful for patients, whereas caregivers might feel relieved from the responsibility of providing care for an acutely unwell patient. ${ }^{3}$ These factors may explain the discrepancy between the treatment appraisals of patients and caregivers. The discrepancy appears greater in the treatment of patients with schizophrenia whose symptoms in the acute phase can be particularly burdensome to caregivers. ${ }^{30}$

The study identified that symptom improvement in patients was the only factor that was important for the way caregivers appraise treatment, and the association between more favourable symptom improvement and more positive treatment appraisal of caregivers was highly significant. Caregivers may observe symptom improvement in patients and conclude that treatment must have been right. Consequently they tend to rate the different aspects of treatment more positively. Another possible explanation is that caregivers' views adequately reflect the quality of the treatment provided in the hospital. If their views are more positive, the treatment is more likely to be effective and lead to symptom improvement. Longitudinal studies with repeated measures of treatment appraisals and symptoms are required to disentangle these two processes. The CAT for caregivers may be a useful tool in such studies. It is brief and simple. The findings of this study suggest that the psychometric properties, i.e. reliability measured as internal consistency and concurrent validity, demonstrated by the association with symptom change, are acceptable, although the high mean score may indicate a ceiling effect limiting the variability captured at the positive end at the scale.

\section{Implications}

Caregivers' generally positive appraisal of treatment in the hospital may facilitate attempts to involve them in treatment planning and delivery during or following involuntary hospital treatment. It should be easier for clinicians to engage with caregivers who have a positive view of the provided treatment than with discontent and disappointed caregivers. The contrast with the negative views expressed by caregivers in qualitative studies on wider aspects of treatment suggests that such attempts should focus on improving communication with caregivers ${ }^{31}$ and the support available to them rather than the hospital treatment for the patient itself.

The findings might also suggest that caregivers actually have a 'point' when appraising the patients' hospital treatment. Taking into consideration the views of caregivers about the quality of the treatment that involuntary patients receive in hospital - and taking those views seriously - might therefore be not only an ethical requirement and a move to involve caregivers as requested by mental health policies, ${ }^{1,2,32}$ but also reveal relevant information. Our results suggest that this applies to the treatment of diagnostically mixed groups as well as specifically to that of patients with schizophrenia and related disorders.

The importance of symptom improvement for caregivers' treatment appraisal held true when the influence of country in this multinational study was adjusted for in the analysis. Thus, the finding seems not to be setting dependent. Nevertheless, the findings should be replicated, and services might consider obtaining caregivers' views of treatment on a routine basis for evaluation. More specific research is required for a better understanding of the processes linking symptom improvement of patients and treatment appraisal of caregivers. Further studies combining quantitative and qualitative methods may explore in more detail what experiences influence caregivers' view of treatment and how patients, caregivers and clinical staff can best share and negotiate their appraisals.
Domenico Giacco, MD, Department of Psychiatry, University of Naples SUN, Naples, Italy and Unit for Social and Community Psychiatry, Barts' and the London School of Medicine and Dentistry, Queen Mary College, University of London, UK; Andrea Fiorillo, MD, PhD, Valeria Del Vecchio, MD, PhD, Department of Psychiatry, University of Naples SUN, Naples, Italy; Thomas Kallert, MD, Park Hospital Leipzig, Department of Psychiatry, Psychosomatic Medicine, and Psychotherapy, Leipzig \& Soteria Hospital Leipzig, Leipzig, and Faculty of Medicine, Dresden University of Technology, Dresden, Germany; Georgi Onchev, MD, PhD, Department of Psychiatry, Medical University Sofia, Bulgaria; Jiri Raboch, MD, Department of Psychiatry, Charles University in Prague, 1st Medical School, Czech Republic: Anastasia Mastrogianni, MD, PhD, Psychiatric Hospital of Thessaloniki, Greece; Alexander Nawka, MD, Department of Psychiatry, Charles University in Prague, 1st Medical School, Czech Republic; Tomasz Hadrys, MD, PhD, Department of Medical School, Czech Republic; Tomasz Hadrys, MD, PhD, Department of
Psychiatry, Medical University, Wroclaw, Poland; Lars Kjellin, Dr MedSc, School of Health and Medical Sciences, Psychiatric Research Centre, Örebro University, Sweden; Mario Luciano, MD, Department of Psychiatry, University of Naples SUN, Naples, Italy; Corrado De Rosa, MD, Mario Maj, MD, PhD, Department of Psychiatry, University of Naples SUN, Naples, Italy; Stefan Priebe, FRCPsych, Unit for Social and Community Psychiatry, Barts' and the London School of Medicine and Dentistry, Queen Mary College, University of London, UK

Correspondence: Domenico Giacco, Department of Psychiatry, University of Naples SUN, Largo Madonna delle Grazie, 80138, Naples, Italy. Email: giaccod@gmail.com

First received 2 Apr 2012, final revision 14 Aug 2012, accepted 17 Aug 2012

\section{Funding}

This work and the multisite research project 'European Evaluation of Coercion in Psychiatry and Harmonization of Best Clinical Practice' (EUNOMIA) were supported by the European Commission - Quality of Life and Management of Living Resources Programme (contract No. QLG4-CT-2002-01036).

\section{Acknowledgements}

The authors gratefully acknowledge the valuable contribution of all the research workers involved in the data collection, the patients and caregivers who participated and the staff of the participating hospitals.

\section{References}

1 World Health Organization. Integrating Mental Health Care into Primary Care: A Global Perspective. WHO, 2008 (http://www.who.int/mental_health/policy/ services/mentalhealthintoprimarycare/en/index.html).

2 Department of Health. National Service Framework for Mental Health Modern Standards and Service Models. Department of Health, 1999 (http://www.dh.gov.uk/en/Publicationsandstatistics/Publications/ PublicationsPolicyAndGuidance/DH_4009598).

3 Jankovic J, Yeeles K, Katsakou C, Amos T, Morriss R, Rose D, et al. Family caregivers' experiences of involuntary psychiatric hospital admissions of their relatives - a qualitative study. PLOS One 2011; 6: e25425.

4 Wilkinson C, McAndrew S. 'I'm not an outsider, I'm his mother!' A phenomenological enquiry into carer experiences of exclusion from acute psychiatric settings. Int J Ment Health Nurs 2008; 17: 392-401.

5 Hoge SK, Lidz C, Mulvey E, Roth L, Bennett N, Siminoff L, et al. Patient, family, and staff perceptions of coercion in mental hospital admission: an exploratory study. Behav Sci Law 1993; 11: 281-93.

6 Hoge SK, Lidz CW, Eisenberg M, Monahan J, Bennett N, Gardner W, et al. Family, clinician, and patient perceptions of coercion in mental hospital admission. A comparative study. Int J Law Psychiatry 1998; 21: 131-46.

7 Ostman M, Hansson L. Appraisal of caregiving, burden and psychological distress in relatives of psychiatric inpatients. Eur Psychiatry 2004; 19: 402-7.

8 Ostman $\mathrm{M}$, Hansson L, Andersson K. Family burden, participation in care and mental health-an 11-year comparison of the situation of relatives to compulsorily and voluntarily admitted patients. Int J SOC Psychiatry 2000; 46: 191-200.

9 Greenwood N, Key A, Burns T, Bristow M, Sedgwick P. Satisfaction with in-patient psychiatric services. Relationship to patient and treatment factors. Br J Psychiatry 1999; 174: 159-63.

10 Kallert TW, Glöckner M, Onchev G, Raboch J, Karastergiou A, Solomon Z, et al. The EUNOMIA project on coercion in psychiatry: study design and preliminary data. World Psychiatry 2005; 4: 168-72.

11 Priebe S, Katsakou C, Glöckner M, Dembinskas A, Fiorillo A, Karastergiou A, et al. Patients' views of involuntary hospital admission after 1 and 3 months: prospective study in 11 European countries. Br J Psychiatry 2010; 196 $179-85$ 
12 Fiorillo A, De Rosa C, Del Vecchio V, Jurjanz L, Schnall K, Onchev G, et al. How to improve clinical practice on involuntary hospital admissions of psychiatric patients: suggestions from the EUNOMIA study. Eur Psychiatry 2011; 26: 201-7.

13 Kallert TW, Katsakou C, Adamowski T, Dembinskas A, Fiorillo A, Kjellin L, et al. Coerced hospital admission and symptom change - a prospective observational multi-centre study. PLOS ONE 2011; 6: e28191.

14 Fiorillo A, Giacco D, De Rosa C, Kallert T, Katsakou C, Onchev G, et al. Patient characteristics and symptoms associated with perceived coercion during hospital treatment. Acta Psychiatr Scand 2012; 125: 460-7.

15 World Health Organization. The ICD-10 Classification of Mental and Behavioural Disorders: Clinical Descriptions and Diagnostic Guidelines. WHO, 1992.

16 Richardson M, Katsakou C, Torres-González F, Onchev G, Kallert T, Priebe S. Factorial validity and measurement equivalence of the Client Assessment of Treatment Scale for psychiatric inpatient care - a study in three European countries. Psychiatry Res 2011; 188: 156-60.

17 Priebe $S$, Katsakou $C$, Amos T, Leese M, Morriss R, Rose D, et al. Patients' views and readmissions 1 year after involuntary hospitalisation. Br J Psychiatry 2009; 194: 49-54.

18 Lukoff D, Nuechterlien K, Ventura J. Manual for the expanded Brief Psychiatric Rating Scale. Schizophr Bull 1986; 13: 261-76.

19 Katsakou C, Priebe S. Patient's experiences of involuntary hospital admission and treatment: a review of qualitative studies. Epidemiol Psichiatr Soc 2007; 16: $172-8$.

20 Katsakou C, Rose D, Amos T, Bowers L, McCabe R, Oliver D, et al. Psychiatric patients' views on why their involuntary hospitalisation was right or wrong: a qualitative study. Soc Psychiatry Psychiatr Epidemiol 2012; 47: 1169-79.

21 Perreault M, Rousseau M, Provencher H, Roberts S, Milton D. Predictors of caregiver satisfaction with mental health services. Community Ment Health J 2012; 48: 232-7.

22 Stengård E, Honkonen T, Koivisto AM, Salokangas RK. Satisfaction of caregivers of patients with schizophrenia in Finland. Psychiatr Serv 2000; 51: 1034-9.
23 Solomon P, Marcenko M. Families of adults with severe mental illness: their satisfaction with inpatient and outpatient treatment. Psychosoc Rehabil J 1992; 16: 121-34.

24 Magliano L, Fadden G, Economou M, Held T, Xavier M, Guarneri M, et al. Family burden and coping strategies in schizophrenia: 1-year follow-up data from the BIOMED I study. Soc Psychiatry Psychiatr Epidemiol 2000; 35: 109-15.

25 Magliano L, Fiorillo A, Fadden G, Gair F, Economou M, Kallert T, et al. Effectiveness of a psychoeducational intervention for families of patients with schizophrenia: preliminary results of a study funded by the European Commission. World Psychiatry 2005; 4: 45-9.

26 Magliano L, Fiorillo A, Malangone C, De Rosa C, Maj M. Patient functioning and family burden in a controlled, real-world trial of family psychoeducation for schizophrenia. Psychiatr Serv 2006; 57: 1784-91.

27 Awad AG, Voruganti LN. The burden of schizophrenia on caregivers: a review. Pharmacoecon 2008; 26: 149-62.

28 Magliano L, Orrico A, Fiorillo A, Del Vecchio H, Castiello G, Malangone C, et al. Family burden in bipolar disorders: results from the Italian Mood Disorders Study (IMDS). Epidemiol Psichiatr Soc 2009; 18: 137-46.

29 Fiorillo A, Bassi M, de Girolamo G, Catapano F, Romeo F. The impact of a psychoeducational intervention on family members' views about schizophrenia: results from the OASIS Italian multi-centre study. Int J SOC Psychiatry 2011; 57: 596-603.

30 Magliano L, Fiorillo A, Rosa C, Maj M. Family burden and social network in schizophrenia vs. physical diseases: preliminary results from an Italian national study. Acta Psychiatr Scand 2006; 429: S60-3.

31 Priebe S, Dimic S, Wildgrube C, Jankovic J, Cushing A, McCabe R. Good communication in psychiatry - a conceptual review. Eur Psychiatry 2011; 26 403-7.

32 Noble LM, Douglas BC, Newman SP. What do patients expect of psychiatric services? A systematic and critical review of empirical studies. Soc Sci Med 2001; 52: 985-98.

\section{poem}

\section{Blackfriars' Bridge}

\section{Gail Campbell}

We were friends, sort-of

Rattling in the nuthouse with Cobain

If you had a quid would you phone?

Take medicine from me:

You made the world better, somehow.

How to say, take care love?

Hey, sharp star

They found you washed up

On the dirty old river shore.

It's a closed nutcracker thing

See, we're here in pieces.

Farewell, brave star

This poem is from Gail Campbell's book How Things Fall, published in 2006 by Survivors' Poetry. Gail Campbell was mentored by Debjani Chatterjee.

Chosen by Femi Oyebode. 